\title{
Transatlantica
}

Revue d'études américaines. American Studies Journal

$2 \mid 2014$

Aesthetics of Theory in the Modern Era and Beyond / Photographie documentaire

\section{Stéphane Vanderhaeghe, Robert Coover \& the Generosity of the Page}

Sylvie Bauer

(2) OpenEdition

Journals

Electronic version

URL: https://journals.openedition.org/transatlantica/7092

DOI: $10.4000 /$ transatlantica.7092

ISSN: 1765-2766

Publisher

Association française d'Etudes Américaines (AFEA)

Electronic reference

Sylvie Bauer, "Stéphane Vanderhaeghe, Robert Coover \& the Generosity of the Page", Transatlantica

[Online], 2 | 2014, Online since 10 March 2015, connection on 02 February 2023. URL: http://

journals.openedition.org/transatlantica/7092 ; DOI: https://doi.org/10.4000/transatlantica.7092

This text was automatically generated on 2 February 2023.

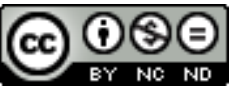

Creative Commons - Attribution-NonCommercial-NoDerivatives 4.0 International - CC BY-NC-ND 4.0

https://creativecommons.org/licenses/by-nc-nd/4.0/ 


\title{
Stéphane Vanderhaeghe, Robert Coover \& the Generosity of the Page
}

\author{
Sylvie Bauer
}

\section{REFERENCES}

VANDERHAEGHE, Stéphane, Robert Coover \& the Generosity of the Page, Champaign, IL, Dalkey Archive Press, 2013, 608 pages, SBN-13 : 9781564788078 , \$35

1 Comment commencer à parler d'un texte qui montre combien l'œuvre de Coover ne commence jamais vraiment, qui dit comment elle s'écrit et se lit par le milieu, comment elle s'évertue à renoncer à tout commencement en rejouant les mêmes partitions? Comment rendre compte d'un ouvrage qui lit le geste critique tout autant que le texte de fiction comme la tentative toujours vouée à l'échec de saisir le sens, de saisir le texte qui s'offre au lecteur avec une générosité sans fin, soulignée par le titre de l'ouvrage de Stéphane Vanderhaeghe? A plusieurs reprises, le lecteur de cet ouvrage critique atypique est confronté au simulacre de sa propre activité : "commenting upon a text by Coover is bound to appear as a platonic simulacrum, inexorably held at a third remove from the text you may catch glimpses of now and then, but can never really seize or lastingly hold between your fingers » (203). Se fait jour alors le geste illusoire et jubilatoire qui consiste à se laisser prendre par une œuvre qui n'a de cesse de se défier du signifié et de mettre en scène la crise du sens, de libérer le réel de la tyrannie du logos, préférant à la possibilité d'une quête herméneutique " un érotisme de l'art, de l'écriture, de la nature ", mettant à mal l'ordre symbolique en exhibant la lettre et la dimension littérale de l'écriture. Toute en variations et en mélodies, l'écriture est chez Coover, une invitation à se laisser tomber dans la langue (« Let yourself drop into language ", 444) en jouant avec les signifiants et l'autonomie de la lettre. Elle est alors événement inlassablement rejoué, inévitablement singulier à chaque lecture.

2 La lecture - car c'est tout autant de cela qu'il est question dans le livre de Stéphane Vanderhaeghe que d'écriture, l'une et l'autre confondues dans le même mouvement - 
résiste tout comme le texte résiste au sens, se dérobant indéfiniment dans un mouvement infini fait de configurations et de reconfigurations, déjouant la ligne, contrant tout sens produit d'avance ou figé tant les textes de Coover, tels des précipités instables, étalent à la surface leur infinie variété et leur instabilité constitutive. "Chemins aux sentiers qui bifurquent» (159), les textes de Robert Coover font converger et diverger les lignes narratives et circuler le sens, enjoignant le lecteur à parcourir tous les sentiers en même temps, des sentiers éminemment mouvants qui ne sauraient être réduits à une ou des ligne(s) : «Reread then, again, 'to celebrat[e] not the line, dull as death itself, but the motion that has made it' (Pinocchio in Venice, 258). Not the traces left on a page, but their relentless tracing and retracing, not the wakes, but the perpetual waking of discarded possibilities " (159). Les romans et nouvelles de Coover et le livre de Stéphane Vanderhaeghe créent des arabesques qui s'effacent au fur et à mesure qu'elles se tracent, écriture sans début ni fin, par le milieu comme dirait Deleuze. Dans les deux cas, la composition est toute musicale, faite de variations, de reprises, de répétitions, de réinventions qui sont autant de seuils et de portes ouvertes, démultipliant les possibles, se refusant à la clôture et préférant le foisonnement: "Writing in the middle, writing of the middle-writing which invents itself as pure middle, constitues itself as an interface of sorts devoid of inside and outside, upside and downside, challenging all hierarchies, discursive, generic, ontological or otherwise, all authority and propriety ( (39). Le résultat est un sentiment de mouvement infini, une redéfinition de la temporalité de la lecture, qui se fait tempo, rythme, mélodie.

$3 \mathrm{Au}$ corpus foisonnant de Coover fait écho le texte bruissant de Stéphane Vanderhaeghe, l'un se fondant dans l'autre dans un geste performatif par lequel le lecteur (de Coover, de Vanderhaeghe) se trouve saisi par l'écriture et devient partenaire d'écriture. Car les positions - lecture, écriture, écriture, lecture - sont tout aussi mouvantes, changeantes et instables que les textes (celui de Vanderhaeghe, ceux de Coover, ceux des critiques et philosophes mentionnés dans l'imposante bibliographie). L'auteur (Coover, Vanderhaeghe) défait plus qu'il ne les brouille, les frontières, les rend poreuses, privilégie les seuils qui sont la promesse renouvelée de l'infini des possibles, ni dedans, ni dehors, milieu encore et toujours. Le texte est alors pluriel, partition jouée par une infinité de mains dans un geste intertextuel qui « invalide la différence entre texte cité et texte citant, texte parodié et parodie » (86, ma traduction). Les textes se rencontrent sur le même "plan d'existence ", engagés dans un dialogue sans fin et sans hiérarchie. Ainsi, la citation est à la fois omniprésente et totalement absente dans Robert Coover \& the Generosity of the Page : l'italique y indique parfois la citation empruntée au corpus de l'écrivain américain, parfois rien d'autre qu'un numéro de page ne vient indiquer qu'il s'agit d'une citation. Le cadre théorique, quant à lui est systématiquement décadré, dégondé de ne pas être enserré par des guillemets, plein d'une parole jamais attribuée à des «auteurs ». La parole et la pensée circulent alors, portant l'écriture à la puissance de l'impersonnel, lui donnant la force d'une adresse sans origine.

4 Geste et performance, l'écriture de Stéphane Vanderhaeghe, comme celle de Robert Coover, s'adresse à un/des destinataire(s) dont l'identité, en fin de compte, importe peu. Entièrement écrit à la deuxième personne, cet ouvrage invite le lecteur à entrer de plain pied dans les plis, replis, déplis de l'écriture et de la lecture, à accepter le corps à corps avec l'écriture, à éprouver le texte comme un flux d'affects, "the life of forces and flows traversing [...] the writing itself » (449). Texte en mouvement servi par une écriture magnifique qui lui donne son souffle et ses rythmes, Robert Coover \& the 
Generosity of the Page est une puissante évocation de l'œuvre de Coover dont les qualités résident autant dans la lecture subtile que l'auteur fait que dans le geste d'une écriture performative.

\section{AUTHORS}

\section{SYLVIE BAUER}

Université Rennes 2 